\title{
Mathematics
}

\section{Beyond reasonable doubt?}

\section{from Ian Stewart}

WHEREAS the mathematical discipline of Number Theory deals with properties of whole numbers, that of Analysis is concerned with the continuous properties of real and complex numbers. Despite this difference, Bernhard Riemann, in 1859, discovered profound connections between the two, leading to what is now known as Analytic Number Theory. Riemann developed a theory of the zeta function, defined for real $s>1$ by

$$
\zeta(s)=1^{-s}+2^{-s}+3^{-s}+\ldots
$$

and for complex $s$ by analytical continuation, and showed how it could be used to study the distribution of prime numbers (Monatsber. Akad. Berlin, 671; 1859). In particular, the number of primes in a given interval could be estimated provided enough was known about the zeros of the zeta function: values $s$ such that $\zeta(s)=0$. There are some relatively obvious zeros at $s=-2,-4,-6, \ldots$. Riemann conjectured that all the remaining zeros lie on the complex line $s=1 / 2+i y$ where $y$ is real. This, the Riemann Hypothesis, if true, has extensive repercussions - not all within Number Theory. It remains an intri- guing and important unsolved problem.

Computer calculations, based on various known features of the zeta function, have shown that Riemann's hypothesis holds good for the first $\mathbf{3 2 0}$ million zeros (Edwards, H.M. Riemann's Zeta Function, Academic, New York; 1974). This would appear to be strong evidence in favour of the hypothesis - but is it? An analogous question is illuminating. In 1885 T.J. Stieltjes came up with a different conjecture which would, if true, imply the Riemann Hypothesis (C. r. hebd. Seanc. Acad. Sci., Paris 101, 368). It was proposed again by F. Mertens in 1897, after whom it is now named (Sber. preuss. Akad. Wiss. 106, 761). It can be stated as follows. For any integer $n$, let $M(n)$ be the difference between the number of integers less than $n$ that are products of an even number of distinct primes and the number that are products of an odd number of primes. The Mertens Conjecture is that $M(n)<\sqrt{n}$ for $n>1$. For example when $\sqrt{n}=16$ there are five numbers in the 'even' case $(1,6,10,14,15)$ and six 'odd' $(2,3,5,7,11,13)$, so $M(16)=1$ which is cer- tainly less than 4 . The Mertens Conjecture has been verified by computer calculations for the first 10 billion values of $n$.

Despite the apparently overwhelming evidence in its favour, it has recently been discovered that the Mertens Conjecture is false. Andrew Odlyzko (Bell Laboratories) and Herman te Riele (Centre for Mathematics and Computer Science, Amsterdam) have shown in unpublished work, based upon fast computer techniques, that there exist infinitely many values of $n$ for which the conjecture fails. The first 'bad' $n$ is no larger than 10 to the power 10 to the power 70 (or thereabouts), an order of magnitude totally inaccessible to direct computation. It is possible that an explicit calculation of the actual 'bad' values would not be an improvement on the demonstration that they must exist.

The Riemann Hypothesis is unaffected by this result: it is still unsolved. Experts have long felt that to prove it by way of the Mertens Conjecture would be 'too easy', and their suspicions are now vindicated. Meanwhile, if the first 10 billion numbers are conspiring to mislead us about the Mertens Conjecture, what faith can we place on a mere 320 million in favour of the Riemann Hypothesis?

Ian Stewart is a Reader in Mathematics at the University of Warwick and currently a visiting Professor at the University of Houston, Texas 77004.

\section{Astronomy}

\section{IRAS circular 11}

THE sources in this circular all have a firm detection at $60 \mu \mathrm{m}$, are at least $20^{\circ}$ away from the galactic plane and have continua representative of 'warm' galaxies. Each search box has been inspected on SERC/ESO Schmidt survey plates or on National Geographic/Palomar Schmidt plates, and contains an obvious candidate, in most cases a galaxy. Selection of sources and the optical search were done by G.K. Miley and M.H.K. de Grijp, Sterrewacht Leiden, The Netherlands. Source names derived as before; see Nature 309, 480; 1983. Position is given at equinox 1950.0. Measurements were made between epochs 1983.1 and 1983.9. In the table, a m denotes arc min.

Also now available, but not to be published in Nature is IRAS circular 10 which lists 179 sources selected from the IRAS minisurvey of an area of approximately 300 square degrees between 9 and 16 February 1983 (see RowanRobinson et al. Astrophys. J. Lett. 278, L7; 1984). The sources satisfied the criteria of being more than $20^{\circ}$ away from the galactic plane, reproducible on successive scans and with a signal-to-noise ratio of greater than $9: 1$ in at least one of the wavelength bands.

\begin{tabular}{|c|c|c|c|c|c|c|c|c|c|c|c|c|c|c|}
\hline \multirow{2}{*}{$\begin{array}{l}\text { Source } \\
\text { IRAS }\end{array}$} & RA & \multirow{2}{*}{$\begin{array}{c}\text { Dec } \\
\text { deg a m }\end{array}$} & \multicolumn{4}{|c|}{ Flux density (Jy) } & \multirow{2}{*}{$\begin{array}{l}\text { Source } \\
\text { IRAS }\end{array}$} & \multicolumn{2}{|c|}{ RA } & \multirow{2}{*}{$\begin{array}{c}\text { Dec } \\
\text { deg a } \mathrm{m}\end{array}$} & \multicolumn{4}{|c|}{ Flux density (Jy) } \\
\hline & $h \min s$ & & $12 \mu \mathrm{m}$ & $25 \mu \mathrm{m}$ & $60 \mu \mathrm{m}$ & $100 \mu \mathrm{m}$ & & h min & $s$ & & $12 \mu \mathrm{m}$ & $25 \mu \mathrm{m}$ & $60 \mu \mathrm{m}$ & $100 \mu \mathrm{m}$ \\
\hline $0425-072 \mathrm{P} 11$ & 042522.2 & -0715 & $<0.4$ & 0.4 & 0.9 & 1.3 & $1320-342 P 11$ & 1320 & 44.8 & -3415 & $<0.4$ & $<0.3$ & 0.6 & $<1.7$ \\
\hline $0425-046 \mathrm{P} 11$ & $04 \quad 2557.1$ & -0440 & $<0.2$ & 1.6 & 4.5 & 4.3 & $1329+022 \mathrm{P} 11$ & 1329 & 19.7 & +0216 & $<0.2$ & $<0.6$ & 1.1 & 1.2 \\
\hline $0428-097 \mathrm{P} 11$ & $04 \quad 28 \quad 11.0$ & -0944 & $<0.3$ & 0.4 & 0.7 & $<1.4$ & $1331-301 \mathrm{P} 11$ & 1331 & 28.9 & -3007 & $<0.2$ & $<0.3$ & 0.8 & $<1.0$ \\
\hline $0432-143 P 11$ & 043232.5 & -1419 & 1.2 & 3.8 & 7.9 & 11.3 & $1331-234 \mathrm{P} 11$ & 1331 & 51.2 & -2325 & $<0.2$ & $<0.4$ & 1.0 & 2.2 \\
\hline $0438-084 \mathrm{P} 11$ & $04 \quad 38 \quad 28.8$ & -0828 & 0.5 & 1.8 & 3.4 & 2.8 & $1331-231 P 11$ & 1331 & 56.4 & $\begin{array}{ll}-23 & 11\end{array}$ & $<0.9$ & $<0.5$ & 1.0 & 2.0 \\
\hline $0450-032 \mathrm{P} 11$ & $\begin{array}{lll}04 & 50 & 14.1\end{array}$ & $-03 \quad 17$ & $<0.6$ & 0.5 & 1.0 & 1.5 & $1333-340 \mathrm{P} 11$ & 1333 & 01.8 & -3402 & 0.4 & 0.7 & 1.2 & 1.5 \\
\hline $0450-184 \mathrm{P} 11$ & $0450 \quad 40.8$ & -1826 & $<0.2$ & $<0.5$ & 0.9 & 2.5 & $1354-203 P 11$ & 1354 & 33.1 & $\begin{array}{ll}-20 & 22\end{array}$ & $<0.6$ & $<0.8$ & 1.5 & 2.5 \\
\hline $0509-024 \mathrm{P} 11$ & $\begin{array}{lll}05 & 09 & 03.8\end{array}$ & -0226 & 0.3 & 1.2 & 2.0 & $<1.5$ & $1356-188 \mathrm{P} 11$ & 1356 & 16.2 & -1848 & $<0.3$ & $<0.7$ & 1.4 & 3.6 \\
\hline $0513-235 \mathrm{P} 11$ & $\begin{array}{lll}05 & 13 & 44.2\end{array}$ & -2331 & $<0.2$ & $<0.5$ & 0.9 & 3.1 & $1402-316 \mathrm{P} 11$ & 1402 & 09.7 & -3140 & $<0.5$ & $<0.3$ & 0.7 & $<1.5$ \\
\hline $0521-122 \mathrm{P} 11$ & 052147.0 & $\begin{array}{ll}-12 & 12\end{array}$ & $<0.2$ & 0.4 & 0.6 & $<2.8$ & $1404+012 \mathrm{P} 11$ & 1404 & 04.9 & +0117 & $<0.2$ & $<0.5$ & 1.1 & $<1.2$ \\
\hline $0531-206 \mathrm{P} 11$ & 053157.0 & -2036 & $<0.2$ & $<0.4$ & 1.2 & 2.0 & $1423-116 P 11$ & 1423 & 27.8 & -1140 & $<0.4$ & $<0.4$ & 0.8 & 1.6 \\
\hline $0556-348 \mathrm{P} 11$ & $05 \quad 56 \quad 31.9$ & -3453 & $<0.7$ & $<0.3$ & 0.5 & $<1.3$ & $1428-030 \mathrm{P} 11$ & 1428 & 51.4 & -0304 & $<0.2$ & $<0.4$ & 1.0 & 2.0 \\
\hline $0611-326 \mathrm{P} 11$ & $\begin{array}{lll}06 & 11 & 30.1\end{array}$ & -3240 & $<0.2$ & 0.4 & 0.9 & 1.5 & $1431-326 \mathrm{P} 11$ & 1431 & 42.8 & $\begin{array}{ll}-32 & 37\end{array}$ & $<0.3$ & 0.4 & 1.0 & $<1.2$ \\
\hline $0815+035 P 11$ & $\begin{array}{lll}08 & 15 & 18.0\end{array}$ & +0331 & $<0.4$ & $<0.3$ & 0.7 & $<1.7$ & $1444-219 P 11$ & 1444 & 35.4 & -2156 & $<0.6$ & $<0.4$ & 1.1 & 1.8 \\
\hline $0818+033 P 11$ & $\begin{array}{lll}08 & 18 & 49.8\end{array}$ & +0319 & $<0.2$ & $<0.4$ & 0.8 & 2.0 & $1458-222 \mathrm{P} 11$ & 1458 & 56.7 & $-22 \quad 15$ & $<0.3$ & $<0.4$ & 0.8 & 2.6 \\
\hline $1036-190 \mathrm{P} 11$ & $1036 \quad 39.5$ & -1904 & $<0.4$ & $<0.3$ & 0.6 & $<1.4$ & $1509-211 \mathrm{P} 11$ & 1509 & 06.6 & -2107 & $<0.4$ & 0.7 & 1.8 & 2.0 \\
\hline $1051-273 P 11$ & $\begin{array}{lll}10 & 51 & 09.1\end{array}$ & -2722 & $<0.2$ & 0.4 & 1.0 & $<1.2$ & $1524+007 \mathrm{P} 11$ & 1524 & 04.5 & +0046 & $<0.2$ & 0.5 & 1.0 & 1.5 \\
\hline $1105-115 \mathrm{P} 11$ & 110548.9 & -1131 & $<0.2$ & 0.4 & 0.8 & $<1.4$ & $1548-037 \mathrm{P} 11$ & 1548 & 03.4 & -0344 & $<0.4$ & 0.8 & 1.3 & $<1.7$ \\
\hline $1119+045 P 11$ & 111955.6 & +0431 & $<0.7$ & 0.5 & 0.9 & 2.7 & $1618+068 \mathrm{P} 11$ & 1618 & 30.1 & +0651 & $<0.3$ & $<0.3$ & 0.7 & $<1.2$ \\
\hline $1121-281 \mathrm{P} 11$ & $\begin{array}{lll}11 & 2133.3\end{array}$ & -2806 & $<0.4$ & 0.4 & 0.7 & $<0.8$ & $1832-594 P 11$ & 1832 & 32.8 & $\begin{array}{ll}-59 & 26\end{array}$ & 0.6 & 1.5 & 3.6 & 5.6 \\
\hline $1246-111 \mathrm{P} 11$ & $1246 \quad 53.3$ & -1107 & $<0.2$ & 0.8 & 1.7 & 2.1 & $1833-654 \mathrm{P} 11$ & 1833 & 21.8 & -6528 & 0.8 & 2.5 & 2.6 & $<1.7$ \\
\hline $1249-131 \mathrm{P} 11$ & 124935.1 & -1308 & $<0.2$ & $<0.5$ & 1.3 & 2.8 & $1840-624 P 11$ & 1840 & 07.9 & -6225 & 0.4 & 1.1 & 2.2 & 4.4 \\
\hline $1304-234 \mathrm{P} 11$ & $1304 \quad 23.5$ & -2324 & 0.4 & 1.3 & 2.6 & 4.1 & $1844-532 P 11$ & 1844 & 14.7 & $\begin{array}{ll}-53 & 12\end{array}$ & $<0.2$ & $<0.4$ & 0.8 & $<1.7$ \\
\hline $1305-241 \mathrm{P} 11$ & $\begin{array}{lll}13 & 05 & 59.1\end{array}$ & -2407 & $<0.2$ & 0.7 & 1.6 & 2.1 & $1919-421 P 11$ & 1919 & 23.9 & -4206 & $<0.4$ & $<0.5$ & 1.1 & $<2.1$ \\
\hline $1315-098 \mathrm{P} 11$ & $13 \quad 15 \quad 31.4$ & -0949 & $<0.2$ & $<0.5$ & 0.9 & $<1.6$ & $1955-140 \mathrm{PII}$ & 1955 & 49.9 & -1405 & 1.0 & 1.5 & 1.3 & 3.1 \\
\hline $1316-242 \mathrm{P} 11$ & 131649.3 & -2413 & $<0.2$ & $<0.4$ & 0.9 & $<2.1$ & $1958-183 P 11$ & 1958 & 02.7 & -1818 & $<0.5$ & $<0.7$ & 1.1 & 1.4 \\
\hline $1319-164 \mathrm{P} 11$ & $\begin{array}{lll}13 & 19 & 42.3\end{array}$ & -1627 & 0.8 & 2.9 & 6.3 & 7.1 & $2037-383 P 11$ & 2037 & 58.7 & -3822 & $<0.4$ & $<0.5$ & 1.5 & $<2.2$ \\
\hline
\end{tabular}

